\title{
MEASURE ESTIMATES, HARNACK INEQUALITIES AND RICCI LOWER BOUND
}

\author{
BY Yu WANG AND XiANGWEN Zhang
}

\begin{abstract}
On the Riemannian metric-measure space, we establish an Alexandrov-Bakelman-Pucci type estimate connecting the Bakry-Émery Ricci curvature lower bound, the modified Laplacian and the measure of certain special sets. We apply this estimate to prove the Harnack inequalities for the modified Laplacian operator (and fully non-linear operators, see the Appendix). These inequalities seem not available in the literature and our proof, based solely on the ABP estimate, does not use standard techniques.
\end{abstract}

1. Introduction. The aim of this paper is to extend the AlexandrovBakelman-Pucci (ABP) techniques to general Riemannian setting and use them to study the relation between Ricci lower bound and elliptic PDEs on Riemannian metric-measure spaces. In particular, we establish an ABP type inequality (Theorem 1.2), which connects the measure of some specific sets contact sets (Definition 1.1) and the Bakry-Émery Ricci curvature. The idea of this work is largely influenced by the remarkable papers by Cabré $[2$ and Savin [18. To illustrate the power of the ABP techniques, we shall consider, on a smooth Riemannian metric-measure space $(\mathscr{M}, g, \nu)$ with $\nu=e^{-V} \operatorname{vol}_{g}$, the modified Laplacian operator

$$
\Delta_{\nu} u=\Delta u-g(\nabla u, \nabla V),
$$

which can also be defined in a more geometric way as $\mathcal{L}_{\nabla u} \nu=\left(\Delta_{\nu} u\right) \nu$, where $\mathcal{L}$ stands for the Lie derivative. We will prove the Harnack inequalities (Theorems 1.3 1.5 for this operator under the assumption on the local lower bound of the Bakry-Émery Ricci curvature. These results are the generalizations of the Harnack inequalities proved by Yau and his collaborators (see [7], [8], 24] ). So

2010 Mathematics Subject Classification. 35J15, 58J05.

Key words and phrases. ABP estimate, Krylov-Safonov Harnack inequality, metricmeasure space, Bakry-Émery Ricci curvature, weighted Laplacian operator, Pucci operator. 
general Harnack inequalities seem to be unavailable in the literature; and does not use standard methods, which rely on the variational structure of Laplacian operator, in geometric analysis.

$\mathrm{ABP}$ techniques are of central importance in the contemporary study of elliptic equations. However, they are not directly applicable in general Riemannian settings. This is due to the fact that non-constant affine function does not exist on general manifolds and the tangent bundles are separated from the underlying spaces. These difficulties were resolved by Cabré [2]. In his pioneer work, Cabré proposed to replace the linear functions by paraboloids-squared distance functions $\rho^{2}(\cdot, y)$ and consider the following special sets:

DEFINITION 1.1. Let $\Omega$ be a bounded open domain in the Riemannian manifold $(\mathscr{M}, g)$ and $u \in C(\Omega)$. For a given $a \geq 0$ and a compact subset $E \subset \mathscr{M}$, the contact set of opening $a$ with vertex set $E$ is defined by

$$
\begin{array}{r}
A(a, E / \Omega, u):=\left\{x \in \bar{\Omega}: \inf _{\bar{\Omega}}\left\{u(\cdot)+\frac{a}{2} \rho^{2}(\cdot, y)\right\}=u(x)+\frac{a}{2} \rho^{2}(x, y)\right. \\
\text { for some } y \in E\},
\end{array}
$$

where $\rho$ is the distance function of the metric $g$. We shall always denote $A(1, E / \Omega, u)$ by $A(E / \Omega, u)$ for convenience and we call $E$ the vertex set.

Using these two new components and replacing the gradient map $\nabla u$ with the map

$$
F[u](x):=\exp _{x}(\nabla u(x)), \quad u \in C^{2}(M),
$$

Cabré was able to control the integral of the Laplacian $\Delta u$ (or more generally non-divergence linear operator, see Definition 1.1 in [2|) over a sub-level set from below by the volume of the domain (see Lemma 4.1 in $[\mathbf{2} \mid$ ). Then following the approach of Krylov-Safonov ([1], [12] $)$, Harnack inequalities for non-divergence equation on Riemannian manifolds with non-negative sectional curvature were derived from this estimate via Calderón-Zygmund decompositions. The approach in $[\mathbf{2}$ was extended by Kim $[\mathbf{1 0}$ by replacing the assumption of non-negative sectional curvature by certain balanced condition on the sectional curvatures according to the given operator $L$ (see condition 4 and 5 in Section 1 of $[\mathbf{1 0}]$ ). In particular, if one considers the Laplacian equation, that condition reduces to the non-negativity of Ricci curvature.

Nevertheless, the power of Cabré's approach has not been fully explored yet. Following his approach, combined with some recent development in the theory of optimal transport ([20 and references therein), we can extend the ABP techniques onto a considerably more general settings. In particular, we prove the following measure estimate formula, which resembles the Euclidean version of $\mathrm{ABP}$ estimate. 
TheOREM 1.2. Let $(\mathscr{M}, g, \nu)$ be a complete Riemannian metric-measure space of dimension $n \geq 2$. Let $E$ be a compact set in $\mathscr{M}$ and $\Omega$ be a bounded open domain. Let $K \in \mathbb{R}$ and $N \in[n, \infty]$ be two constants. Let $u \in C^{2}(\bar{\Omega})$. If $A(E / \Omega, u) \subset \Omega$, then the following statements hold:

(i) If $N<\infty$, then

$$
\operatorname{Ric}_{N, \nu} \geq K g \Rightarrow \nu[E] \leq \int_{A(E / \Omega, u)}\left\{\mathcal{D}_{K, N}[u](x)\right\}^{N} \nu(d x),
$$

where for any $x \in A(E / \Omega, u)$,

$$
\mathcal{D}_{K, N}[u](x):= \begin{cases}\cos \left(\sqrt{\frac{K}{N}}|\nabla u|\right)+\frac{\Delta_{\nu} u}{\sqrt{K N}|\nabla u|} \sin \left(\sqrt{\frac{K}{N}}|\nabla u|\right) & K|\nabla u|>0, \\ 1+\Delta_{\nu} u / N & K|\nabla u|=0 \\ \cosh \left(\sqrt{\frac{-K}{N}}|\nabla u|\right)+\frac{\Delta_{\nu} u}{\sqrt{-K N}|\nabla u|} \sinh \left(\sqrt{\frac{-K}{N}}|\nabla u|\right) & K|\nabla u|<0 .\end{cases}
$$

(ii) If $N=\infty$, then

$$
\operatorname{Ric}_{\infty, \nu} \geq K g \Rightarrow \nu[E] \leq \int_{A(E / \Omega, u)} \exp \left\{-\frac{1}{2} K|\nabla u(x)|^{2}+\Delta_{\nu} u(x)\right\} \nu(d x) .
$$

The upshot of the above formula is that the integral is only calculated on a special set - the contact set, and the lower bound of this integral can be controlled. This is the essence of the Euclidean ABP estimate. Unlike the ABP estimate in Euclidean space, the above formula does not involve infima of the unknown function. However, we shall see this would not limit its application.

We have assumed the underlying manifold is smooth and $u$ is $C^{2}$ near $A(E / \Omega, u)$. In fact, we only need $u$ to be semi-concave (see Definition 16.4, p. 429 in $[20]$ ) near $A$. However, to avoid heavy formulations and to better present the main ideas, we shall stay with $C^{2}$-functions. The underlying idea of proving the above theorem is indeed contained in [2] and consists in applying the area formula to the map $F[u]$ defined by (1.1) on the contact set $A$. Here, rather than use the direct calculation for the Jacobi determinant of $F[u]$ given in $\sqrt{2}$, we employ an ODE comparison estimate proposed in Chapter 14 of 20]. Besides allowing us to establish an estimate for very general curvature condition, this ODE estimate matches in a remarkable way the fine structure of the contact sets (see Lemma 3.6 and Lemma 4.6).

TheOREm 1.3. Let $(\mathscr{M}, g, \nu)$ be a complete smooth Riemannian metricmeasure space. Let $K \geq 0$ and $N \in[n, \infty)$. Let $u \in C^{2}\left(B_{2 R}\right) \cap C\left(\bar{B}_{2 R}\right)$ and $f \in C\left(B_{2 R}\right)$. Suppose

$$
\left.\operatorname{Ric}_{N, \nu}\right|_{B_{2 R}} \geq-K g, \quad \Delta_{\nu} u \leq f \text { in } B_{2 R}, \quad u \geq 0 \text { in } B_{2 R} .
$$


Then

$$
\left(f_{B_{R / 2}} u^{p_{0}}\right)^{1 / p_{0}} \leq C_{0}\left\{\inf _{B_{R / 2}} u+R^{2}\left(f_{B_{2 R}}|f|^{N \eta}\right)^{1 /(N \eta)}\right\},
$$

where $p_{0}, C_{0}$ are constants depending on $\sqrt{K} R, N$ only. Moreover $C_{0}=e^{2 / p_{0}}$ and $\eta:=\eta_{2 R}:=1+\frac{8 R}{\log 2} \sqrt{\frac{K}{N}}$.

TheOREM 1.4. Let $(\mathscr{M}, g, \nu)$ be a complete smooth Riemannian metricmeasure space. Let $K \geq 0$ and $N \in[n, \infty)$. Let $u \in C^{2}\left(B_{2 R}\right) \cap C\left(\bar{B}_{2 R}\right)$ and $f \in C\left(B_{2 R}\right)$. Suppose

$$
\left.\operatorname{Ric}_{N, \nu}\right|_{B_{2 R}} \geq-K g, \quad \Delta_{\nu} u \geq f \text { in } B_{2 R} .
$$

Then, for any $p>0$

$$
\sup _{B_{R / 2}} u \leq C_{1}(p)\left\{\left(f_{B_{R}}\left(u^{+}\right)^{p}\right)^{1 / p}+R^{2}\left(f_{B_{2 R}}|f|^{N \eta}\right)^{1 /(N \eta)}\right\},
$$

where $C_{1}(p)$ is a constant depending on $\sqrt{K} R, N$ and $p$ only.

TheOrem 1.5. Let $(\mathscr{M}, g, \nu)$ be a complete smooth Riemannian metricmeasure space. Let $K \geq 0$ and $N \in[n, \infty)$. Let $u \in C^{2}\left(B_{2 R}\right) \cap C\left(\bar{B}_{2 R}\right)$ and $f \in C\left(B_{2 R}\right)$. Suppose

$$
\left.\operatorname{Ric}_{N, \nu}\right|_{B_{2 R}} \geq-K g, \quad \Delta_{\nu} u=f \text { in } B_{2 R}, \quad u \geq 0 \text { in } B_{2 R} .
$$

Then

$$
\sup _{B_{R / 2}} u \leq C_{2}\left\{\inf _{B_{R / 2}} u+R^{2}\left(f_{B_{2 R}}|f|^{N \eta}\right)^{1 /(N \eta)}\right\}
$$

where $C_{2}$ is a constant depending on $\sqrt{K} R$ and $N$ only.

REMARK 1.6. In the case $K=0$, the integral expressions of the righthand side function $f$ reduce to the standard averaged $L^{N}$-norm and constants $p_{0}, C_{1}, C_{2}, \eta$ are independent of $R$. This agrees with the Harnack inequalities in $[2]$ and $[\mathbf{1 0}]$ when $K=0$ and $\nu=\operatorname{vol}_{g}$. Increasing the exponent of integration by a factor depending on the lower bound of Ricci curvature is necessary. This can easily be seen from the examples in $(K, N)$-Hyperbolic space.

Besides proving Harnack inequalities under more general assumptions, we provide a different presentation of Krylov-Safonov argument in proving Harnack inequalities. In this presentation, the Calderón-Zygmund decomposition (used in [2] is replaced with Vitali's covering lemma. Though it essentially follows the argument in $[\mathbf{2}$, the argument here seems more elementary and 
transparent. It is similar to the covering argument used by Caffarelli in his breakthrough work on real Monge-Ampére equations 4 and by Savin [18.

Harnack inequalities for divergent equations have intensively been studied in geometric analysis. An incomplete list may start with Yau's penetrating work on developing a maximum principle method to prove that complete manifolds with nonnegative Ricci curvature must have a Liouville property [24. His argument was later localized in his joint paper with Cheng $[\mathbf{8}$ and resulted in a gradient estimate for a rather general class of elliptic equations. Yau's method has later been employed by many authors in the study of differential equations on manifolds. In particular, L. Saloff-Coste observed that, using the results of Varopoulos [19, optimal Sobolev inequalities on balls can easily be obtained from the gradient estimate of Cheng-Yau. Based on these Sobolev inequalities and following the approach in $\mathbf{7}]$ and $[\mathbf{6}$, he applied Moser's iteration to prove Harnack inequalities for divergent operators on manifolds with standard Ricci curvature bounded from below, in the case when the right-hand side $f=0$. These results have also been extended to various general cases. For example, in [21, 25], the authors studied the gradient estimate for $p$-harmonic function on Riemannian manifolds. Recently, Li [13] (see also [1]) followed the main line of $[\mathbf{8}$ and obtained the Harnack inequality for solutions of the modified Laplacian equation on Riemannian metric-measure space.

The paper is organized as follows: In Section 2, we fix our notations and conventions. In particular, we give a full list of constants involved in the later proof. Section 3 and Section 4 are devoted to a study of the contact sets and the Jacobi determinant of $d F[u]$, respectively. In these two sections, we shall see how the contact sets, the Jacobi fields and the underlying geometry interact with one another. In Section 5, we prove the measure estimate formula - Theorem 1.2. Section 6 and Section 7 contain some preparations for the proof of Harnack inequalities (Theorems 1.3 1.5). Section 8 contains the main technical lemma used in proving these theorems. In Section 9 and Section 10, the proofs of Theorems 1.3 1.5 are given. In the Appendix, we extend the method proposed in this paper to prove the Harnack inequalities for fullynonlinear uniform elliptic operators on Riemannian manifolds.

2. Notations, Conventions and Constants. In order to avoid any potential confusion, we first state our conventions and notations.

- Riemannian metric-measure space: In the paper, the background manifold is the Riemannian metric-measure space $(\mathscr{M}, g, \nu)$, where $g$ is the Riemannian metric on $\mathscr{M}$ and $\nu=e^{-V}$ vol is a reference measure with $V: M \rightarrow \mathbb{R}$ a $C^{2}$ function. Notice that, if $V=0$, then $\nu$ is just the standard volume measure $\operatorname{vol}_{g} . n \geq 2$ is always used to denote the dimension of $\mathscr{M}$. 
- Curvatures: Recall the definitions of Riemannian curvature tensor

$$
\operatorname{Riem}(X, Y):=D_{Y} D_{X}-D_{X} D_{Y}+D_{[X, Y]}, \quad X, Y \in T \mathscr{M}
$$

and of Ricci curvature

$$
\operatorname{Ric}_{x}(Z):=\sum_{i} g_{x}\left(\operatorname{Riem}\left(Z, e_{i}\right) Z, e_{i}\right), \quad Z \in T_{x} \mathscr{M},
$$

where $e_{i}$ is the orthogonal basis w.r.t. $g$. We also recall the following definition:

Definition 2.1. The Bakry-Émery Ricci curvature associated with $\nu$ with effective dimension $N \in[n, \infty]$ is defined by

$$
\operatorname{Ric}_{N, \nu}= \begin{cases}\operatorname{Ric} & N=n \\ \operatorname{Ric}+D^{2} V-\frac{D V \otimes D V}{N-n} & N>n \\ \operatorname{Ric}+D^{2} V & N=\infty\end{cases}
$$

Here, we assume $V=0$ whenever $N=n$.

For the importance and the geometry of the Bakry-Émery Ricci curvature, one may refer to $\mathbf{1 4}, \mathbf{2 0}, \mathbf{2 3}$ and the references therein.

- Cut-locus: We recall the definition of cut-points and focal points. We follow the conventions in $2 \mathbf{2 0}$ (p. 193). Note that this convention may differ from the one in other texts, but this will not affect the generality of this paper.

Definition 2.2. Fix $x \in(\mathscr{M}, g)$, a point $y$ is called a cut point of $x$ if there is a geodesic $\gamma(t)$ such that $\gamma(0)=x$ and $\gamma\left(t_{c}\right)=y$ and satisfies: i) $\gamma(t)$ is minimizing for all $t \in\left[0, t_{c}\right)$ and ii) $\gamma\left(t_{c}+\epsilon\right)$ is not minimizing for any $\epsilon>0$.

Two points $x$ and $y$ are said to be focal (or conjugate) if $y$ can be written as $\exp _{x}(t W), W \in T_{x} \mathscr{M}$, and the differential $\left.d\right|_{W} \exp _{x}(t \cdot)$ is not invertible.

Given a point $x \in \mathscr{M}$, the cut-locus $C u t(x)$ of $x$ is the set consisting of all cut-points and focal (conjugate) points of $x$.

- Contact relations: We recall the following terminology:

Definition 2.3. Let $\Omega$ be a subdomain of $\mathscr{M}$. Let $u$ and $\varphi$ be two continuous functions defined in $\Omega$. Let $x_{0} \in \Omega$ and $U$ be a subset (not necessarily open) of $\Omega$. We say $\varphi$ touches $u$ from above (resp. below) at $x_{0}$ in $U$ if $\varphi(x) \geq u(x)($ resp. $\varphi(x) \leq u(x))$ for all $x \in U$ and $\varphi\left(x_{0}\right)=u\left(x_{0}\right)$.

We say $\varphi$ touches $u$ from above (resp. below) at $x_{0}$ if there is a neighborhood $U$ of $x_{0}$ such that $\varphi$ touches $u$ from above (resp. below) at $x_{0}$ in $U$.

- Convention in notations:

i) Throughout this paper, a letter $C$, without any subscript, represents a pure constant greater than 1. It might change from line to line. However, we emphasize that it does not depend on any parameter. Moreover, to 
make the proof more transparent, we shall try to use $C$ scarcily and be explicit.

ii) We always mean $B_{r}(x)$ to be the geodesic ball of radius $r$ centered at $x$. We shall omit the center $x$ when it has no particular importance and does not cause any confusion.

iii) Throughout the paper, integrations are performed against the measure $\nu$; and the distance function is denoted by $\rho$. The notation $\rho_{y}$ means the distance from a fixed point $y$.

- Special functions and notations: Herein below, in particular in the proof of the main theorems, numerous parameters and functions appear. In order to give a clear presentation, we shall use several short-hand symbols. Here, we list these notations and some basic facts regarding them.

i) Let $K \geq 0, N \in[n, \infty)$ and $r>0$. We denote

$$
\omega:=2 \sqrt{\frac{K}{N}}, \quad \mathscr{D}_{r}:=2^{N} e^{4 r \sqrt{N K}}, \quad \eta_{r}:=\frac{\log \mathscr{D}_{r}}{N \log 2}=1+\frac{4 r}{\log 2} \sqrt{\frac{K}{N}} .
$$

Here, $\mathscr{D}_{r}$ is introduced to control the local volume doubling constants for metric-measure spaces with Ric $\geq-K g$ used in later sections.

ii) Let $t \in[0, \infty)$, define

$$
\mathcal{H}(t):=t \operatorname{coth}(t), \quad \mathcal{S}(t):=\frac{\sinh (t)}{t} .
$$

Note that $\mathcal{H}, \mathcal{S}$ are differentiable and have a positive derivative for all $t>0 ; \mathcal{H}(0)=\mathcal{S}(0)=1$ by taking limit. Moreover, $\mathcal{S}(t) \cdot \mathcal{H}(t)=\cosh (t)$. Another useful observation here is that $\mathcal{H}(t) \leq 1+t$ for $t \geq 0$.

iii) Let $q \geq 1$ be a constant and $f$ be a continuous function. We denote

$$
\mathcal{I}\left(f, B_{r}, q\right):=r^{2}\left(f_{B_{r}}|f(x)|^{N q} \nu(d x)\right)^{1 /(N q)} .
$$

Properties of this integral are given in Section 7 .

- Constants in the proofs: The following constants will be used frequently in the proof of Harnack inequalities (Sections 8 10). They are fixed for the entire paper. We also provide some rough estimates for them. Denote, for $r>0$

$$
\alpha:=N \mathcal{H}(r \omega), \quad \mu:=\left[4 \alpha^{2}(18)^{\alpha+3} N \cosh (r \omega)\right]^{-N} \mathscr{D}_{2 r}^{-4}, \quad M:=2 \alpha^{2}(18)^{\alpha} .
$$

where $\omega$ and $\mathscr{D}_{r}$ are the constants introduced in (2.3). Note that, by the definition of $\mathcal{H}$, we can estimate $\alpha$ as

$$
N \leq \alpha \leq N+2 r \sqrt{K N} .
$$


We also introduce

$$
p_{0}=\frac{\log \frac{e}{[1+(e-1)(1-\mu)]}}{\log M}
$$

and

$$
\delta_{0}:=\left(2 \mathscr{D}_{2 r}^{4 / N} \cdot \mathcal{S}(\omega r)\right)^{-1}, \quad C_{3}:=4 \mathscr{D}_{2 r}\left(\frac{M^{p_{0}}}{\mu}\right)^{1 / N}, \quad p_{1}:=p_{0} /\left(N \eta_{r}\right)
$$

The following lemma is easy to check.

Lemma 2.4. $\mathscr{D}_{2 r} \frac{M}{\mu^{1 / p_{0}}}\left(\frac{4}{C_{3}}\right)^{N \eta_{r} / p_{0}}<1$; and there exists a pure constant $C$ independent of any parameter such that $\mu, \delta_{0}, 1 / M$ are all greater than $e^{-C\left(r \sqrt{K N}+N^{2}\right)}$.

Proof. The first claim follows directly from the definition of $C_{3}$ and the fact that $\eta_{r}>1$. More precisely, using the definition of $C_{3}$, we compute

$$
\mathscr{D}_{2 r} \frac{M}{\mu^{1 / p_{0}}}\left(\frac{4}{C_{3}}\right)^{N \eta_{r} / p_{0}}=\mathscr{D}_{2 r}^{1-\frac{N \eta_{r}}{p_{0}}} \cdot\left(\frac{M}{\mu^{1 / p_{0}}}\right)^{1-\eta_{r}}<\mathscr{D}_{2 r}^{1-\frac{N \eta_{r}}{p_{0}}}
$$

since $\eta_{r}>1$ and $\frac{M}{\mu^{1 / p_{0}}}>1$ by the definition of $M$ and $\mu$. Next, we note that $D_{2 r}>1$ and $N \eta_{r}>p_{0}$. Then, the first claim follows.

The second claim can be proved directly by using (2.7).

Now we shall give explicit forms of $C_{0}, C_{1}\left(p_{0}\right), C_{2}$ in the statement of Theorems 1.3 1.5. In later proofs, it will be clear that it suffices to choose such constants. We shall also give some rough estimates for these constants.

Lemma 2.5. Define $C_{0}, C_{1}\left(p_{0}\right), C_{2}$ as follows

$$
C_{0}:=e^{2 / p_{0}}, C_{2}=C_{1}\left(p_{0}\right):=\left(3 C_{3} \sum_{k=0}^{\infty} \frac{1}{(1+1 / M)^{k p_{1}}}\right)^{1 / p_{1}} \frac{1}{\delta_{0}},
$$

where $p_{1}$ and $\delta_{0}$ are constants given in (2.8). Then, the following statements hold

i) $1+\left(M^{p_{0}}-1\right) \sum_{k=0}^{\infty}\left(M^{p_{0}}(1-\mu)\right)^{k}=e$;

ii) $e^{1 / p_{0}} \geq \delta_{0} \geq 1$

iii) The constants $p_{0}, C_{0}, C_{1}\left(p_{0}\right), C_{2}$ satisfy

$$
p_{0} \geq \frac{\mu}{4 \log M} \geq e^{-C\left(r \sqrt{K N}+N^{2}\right)}, \quad \max \left\{C_{0}, C_{2}\right\} \leq \exp \left[e^{C\left(r \sqrt{K N}+N^{2}\right)}\right] .
$$

Proof. i) can be verified directly. To show ii), note the relation $\frac{x}{e} \leq$ $1-\log [e-x] \leq \frac{x}{e-1}$, we have then $\frac{1}{p_{0}} \geq \log M / \mu$. By the choice of $M, \mu, \delta_{0}$, it is clear that ii) holds. 
To prove iii), use the previous inequality of log, it is then easy to show $p_{0}$ satisfies the required estimate. Finally, denote $b:=((M+1) / M)^{p_{1}}$, then it is easy to check

$$
\begin{aligned}
\sum_{k=0}^{\infty}\left(\frac{M}{M+1}\right)^{p_{1} k} & =\frac{1}{2}+\frac{1}{2} \operatorname{coth}\left[\frac{1}{2} \log (b)\right]=\frac{1}{2}+(\log b)^{-1} \mathcal{H}\left(\frac{1}{2} \log (b)\right) \\
& \leq 1+\frac{1}{p_{1} \cdot \log (1+1 / M)} .
\end{aligned}
$$

Next, we notice that $\frac{x-1}{e-1} \leq \log x$ for $x \in(1, e)$. It follows that

Therefore,

$$
\log (1+1 / M) \geq \frac{1 / M}{e-1}
$$

$$
\sum_{k=0}^{\infty}\left(\frac{M}{M+1}\right)^{p_{1} k} \leq 1+\frac{(e-1) M}{p_{1}}<\frac{3 M}{p_{1}} .
$$

Then, we can then easily estimate $C_{2}=C_{1}\left(p_{0}\right)$ as stated.

3. Contact Set. In this section, we investigate some properties of the contact set (recall Definition 1.1), in particular the behavior of the unknown function $u$ on its associated contact set. We will see that the contact set recognizes the underlying metric geometry in an elegant way. First, we provide an alternative characterization of the contact set, given in $[\mathbf{2}]$.

Definition 3.1. The concave paraboloid $P_{a, y}$ with vertex $y$ and opening $a$ is a function of the form

$$
P_{a, y}:=-\frac{a}{2} \rho^{2}(x, y)+c_{y}, \quad c_{y}, a \in \mathbb{R}, a \geq 0 .
$$

Similarly, one can define the convex paraboloid.

Proposition 3.2. Let $\Omega \subset \mathcal{M}$ be a bounded domain and $u \in C(\bar{\Omega})$. Assume that $E \subset \Omega$ is closed and $a \geq 0$. Then $x \in A(a, E / \Omega, u)$ if and only if there exists a concave paraboloid $P_{a, y}$ of opening a and vertex $y \in E$ that touches $u$ in $\bar{\Omega}$ from below.

Proof. It immediately follows from the definitions (Definition 1.1, Definition 2.3 and Definition 3.1.

The following proposition contains some basic properties of the contact set (see $[\mathbf{1 8})$. Its proof is a routine check and hence is omitted.

Lemma 3.3. Let $\Omega \subset \mathcal{M}$ be a bounded domain and $u \in C(\bar{\Omega})$. Assume that $E \subset \mathcal{M}$ is a compact subset, then

a) For all $a \geq 0, A(a, E / \Omega, u)$ is closed (hence $\nu$-measurable). 
b) If $u_{k} \in C(\bar{\Omega})$ and $u_{k} \rightarrow u$ uniformly in $\Omega$, then

$$
\limsup _{k \rightarrow \infty} A\left(a, E / \Omega, u_{k}\right)=\bigcap_{j=1}^{\infty} \bigcup_{k \geq j} A\left(a, E / \Omega, u_{k}\right) \subset A(a, E / \Omega, u) .
$$

c) If $a_{k} \rightarrow 0$, then

$$
\limsup _{k \rightarrow \infty} A\left(a_{k}, E / \Omega, u\right)=\bigcap_{j=1}^{\infty} \bigcup_{k \geq j} A\left(a_{k}, E / \Omega, u\right) \subset A(0, E / \Omega, u) .
$$

d) If $E \subset F$, then

$$
A(a, E / \Omega, u) \subset A(a, F / \Omega, u) .
$$

To examine the interaction between contact sets and the underlying metric geometry, we shall need the following notion and proposition from standard Riemannian geometry. We first recall the hessian bound in support sense introduced in [5] (also see [16]).

Definition 3.4. Let $w \in C(\Omega)$. We say $D^{2} w \geq \beta g, \beta \in \mathbb{R}$, in support sense at $x_{0}$ if for every $\epsilon>0$ there exists a smooth function $\varphi_{\epsilon}$ defined in a neighborhood of $x_{0}$ such that

i) $\varphi_{\epsilon}$ touches $w$ from below at $x_{0}$;

ii) $D^{2} \varphi_{\epsilon}\left(x_{0}\right) \geq(\beta-\epsilon) g\left(x_{0}\right)$.

Similarly, one can define $D^{2} w \leq \beta g$ in support sense.

We remark that the condition ii) in the above definition is different from the definition of viscosity solution in PDE. The following well-known property of the distance function (see [16, p. 342) will be used in our proof.

Proposition 3.5. Let $(\mathscr{M}, g)$ be a smooth Riemannian manifold. Given any $y \in \mathscr{M}$, denote the distance function starting from $y$ by $\rho_{y}(\cdot)$. Then $\nabla^{2} \rho_{y}^{2}$ is locally bounded above in support sense, that is, for any compact set $Z$, there exists a constant $Q$ (depending on $\operatorname{diam}(Z)$ and the sectional curvature lower bound over $Z$ ), such that $D^{2} \rho_{y}^{2}(x) \leq Q g$ for any $x \in Z$ in support sense.

The following lemma contains a key property of contact sets.

Lemma 3.6. Let $u \in C(\bar{\Omega})$ and $E \subset \Omega$ be closed. Let $a>0$. Suppose $A(a, E / \Omega, u) \subset \Omega$ and $u$ is locally bounded above in support sense in $\Omega$. Then the following statement holds: if $y \in E$ and the paraboloid $P_{a, y}$ touches $u$ at $x \in A(a, E / \Omega, u)$ from below, then $x$ and $y$ are neither cut-points nor focal points for each other and hence $P_{a, y}$ is smooth at $x$.

Proof. From Proposition 3.5, we know that $\frac{a}{2} \rho_{y}^{2}(x)$ is locally bounded from above in the support sense and therefore $P_{a, y}$ is locally bounded from 
below in the support sense. On the other hand, we assume that $u$ is locally bounded above in support sense and $P_{a, y}$ touches $u$ from below at $x$; therefore $P_{a, y}$ is locally bounded from above in the support sense. Therefore, by the definition of boundedness in support sense, there are two smooth functions $\varphi^{+}, \varphi_{-}$such that $\varphi^{+}$touches $P_{a, y}$ from above at $x$ and $\varphi_{-}$touches it from below at $x$. It follows immediately that $P_{a, y}$ is differentiable at $x$. By the standard Riemannian geometry, $x$ and $y$ are not cut-points of each other.

To show $x, y$ are not focal points to each other, we consider the limit of the second order increment quotient

$$
\Delta^{2} P_{a, y}(x):=\limsup _{|W| \rightarrow 0} \frac{P_{a, y}\left(\exp _{x} W\right)+P_{a, y}\left(\exp _{x}(-W)\right)-2 P_{a, y}(x)}{|W|^{2}} .
$$

The existence of $\varphi^{+}, \varphi_{-}$shows that $\left|\Delta^{2} P_{a, y}(x)\right|<\infty$. By Proposition 2.5 in [15], this eliminates the possibility that $x, y$ are focal points to each other.

Next lemma relates contact sets, the sub-level sets of $u$ and the domain. Such a statement has indeed been used in $[\mathbf{2}]$.

Lemma 3.7. Let $u \in C\left(\bar{B}_{r}\left(x_{0}\right)\right)$. Suppose $u\left(y_{0}\right)=\ell$ for some $y_{0} \in \bar{B}_{r / 2}\left(x_{0}\right)$ and $u \geq t$ in $B_{r}\left(x_{0}\right) \backslash B_{5 r / 6}\left(x_{0}\right)$. If $\ell<t$, then for any $a>0$

$$
A\left(a, \bar{B}_{r / 6}\left(y_{0}\right) / B_{r}\left(x_{0}\right), u\right) \subset B_{5 r / 6}\left(x_{0}\right) \cap\left\{u \leq \ell+\frac{a r^{2}}{36}\right\} .
$$

Proof. Let $P_{a, z}$ be a polynomial touching $u$ at $x_{1}$ for some $z \in B_{r / 6}\left(y_{0}\right)$. By the contact relation, we have

$$
u\left(y_{0}\right) \geq P_{a, z}\left(y_{0}\right)=-\frac{a}{2} \rho^{2}\left(y_{0}, z\right)+\frac{a}{2} \rho^{2}\left(x_{1}, z\right)+u\left(x_{1}\right) .
$$

Thus, we immediately have

$$
u\left(x_{1}\right) \leq P_{a, z}\left(y_{0}\right)+\frac{a}{2} \rho^{2}\left(y_{0}, z\right) \leq \ell+\frac{a r^{2}}{36} .
$$

Therefore, it suffices to show $x_{1} \in B_{5 r / 6}\left(x_{0}\right)$. Suppose on the contrary that $x_{1} \in B_{r}\left(x_{0}\right) \backslash B_{5 r / 6}\left(x_{0}\right)$. Then, by $y_{0} \in B_{r / 2}\left(x_{0}\right)$ and $z \in B_{r / 6}\left(y_{0}\right)$, we know $\rho\left(x_{1}, z\right) \geq \frac{r}{6}$. Thus $\rho^{2}\left(x_{1}, z\right)-\rho^{2}\left(y_{0}, z\right) \geq 0$. However, 3.1) implies

$$
\frac{a}{2}\left(\rho^{2}\left(x_{1}, z\right)-\rho^{2}\left(y_{0}, z\right)\right) \leq u\left(y_{0}\right)-u\left(x_{1}\right) \leq \ell-t<0 .
$$

This is a contradiction.

REMARK 3.8. While sufficient for the purposes of this paper, this is not the most precise relation between contact sets and sub-level sets. However, the proof of above lemma suggests how one could control the relative position of contact sets, sub-level sets and domain. On space with a special feature in metric geometry, such as Euclidean space where the parallelogram law holds, 
very precise relation can be drawn regarding the relative location of contact sets with different opening (see $\mathbf{1 8}]$ ).

4. Jacobi Equation and Jacobi Determinant. In this section, we quote, after [20], some important results regarding the Jacobi equation and its geometrical implications. They are of fundamental importance in developing our ideas. In particular, we shall see how the contact sets match with the Jacobi determinant (Proposition 4.6). The content of this section follows closely Chapter 14 (pp. 365-372 and 379-383) in [20 and its third Appendix (pp. 412-418). In the sequel, we shall always assume the time interval to be $[0,1]$.

Definition 4.1. Let $R: t \rightarrow R(t)$ be a continuous map defined on $[0,1]$, valued in the space of $n \times n$ symmetric matrices. The Jacobi equation associated to $R(t)$ is the following ODE

$$
\ddot{J}(t)+R(t) \cdot J(t)=0 .
$$

for a time-dependent matrix $J: t \rightarrow J(t)$. Such a solution $J$ will be called a Jacobi matrix.

The following propositions contain the main properties of the Jacobi equation which support our reasoning (see pp. 429-432 in [20 for a proof).

Proposition 4.2. Let $J_{0}^{1}$ and $J_{1}^{0}$ be Jacobi matrices defined by the initial conditions

$$
J_{0}^{1}=\dot{J}_{1}^{0}=I, \quad \dot{J}_{0}^{1}=J_{1}^{0}=0 .
$$

Assume $J_{1}^{0}$ is invertible for all $t \in(0,1]$. Then $S(t):=\left[J_{1}^{0}(t)\right]^{-1} J_{0}^{1}(t)$ is symmetric for all $t \in(0,1]$, and it is a decreasing function of $t$, that is $\langle S(t) w, w\rangle$ is decreasing for any vector $w$.

REMARK 4.3. The original statement in the book [20] also states that $S(t)$ is positive for all $t \in[0,1)$. We have confirmed with the author that the latter statement is merely a typo. Indeed, the material in the third Appendix to Chapter 14 in 20] does not rely on positivity of $S(t)$.

Proposition 4.4. Let $S(t)$ be the matrix defined in Proposition 4.2. Let $J(t)$ be a Jacobi matrix satisfying the initial conditions

$$
J(0)=I, \quad \dot{J}(0) \text { is symmetric }
$$

Then, $\dot{J}(0)+S(1) \geq 0$ if and only if $\operatorname{det} J(t)>0$ for all $t \in[0,1)$.

Now, we relate the above pure ODE results to some geometry of Jacobi fields on Riemannian manifolds. The following discussion follows closely [20]. Let $(\mathscr{M}, g)$ be a Riemannian manifold. Given a geodesic $\gamma(t)$, one may parallelly transport an orthonormal frame $e(0)$ at $T_{\gamma(0)} \mathscr{M}$ along $\gamma(t)$ to obtain 
a frame $e(t)$ at $T_{\gamma(t)} \mathscr{M}$. Then, a family of sections $H(t) \in \operatorname{Sym} T_{\gamma(t)} \mathscr{M}$ can be canonically identified with a family of symmetric matrices parametrized by $t$. In the rest of this paper, we shall always use this identification whenever necessary. The eigenvalues of $H(t)$ are independent of choice of the frame $e(t)$. Consider the flow $F[u](t, \cdot)$ defined as follows:

Definition 4.5. Let $u \in C^{2}(\Omega)$. Define

$$
F[u](t, \cdot): \Omega \rightarrow \mathscr{M}, x \mapsto \exp _{x}(t \nabla u(x))
$$

For our convenience, we shall denote $F[u]=F[u](1, \cdot)$; and the symbols $F_{t}[u]$ and $F[u](t, \cdot)$ will be used interchangeably. We also denote the Jacobi transformation $d F[u]$ by $J[u]$.

The next proposition contains some geometric implications of the previous two propositions (see the discussion on pp. 413-414 in [20]).

Proposition 4.6. Let $x, y \in \mathscr{M}$. Suppose $x, y$ are neither cut-points nor focal points to each other. Then the following statements hold:

i) $J[u](t, x)$ is a smooth (w.r.t. time $t)$ Jacobi matrix associated to

$$
R_{i j}(t, x)=\operatorname{Riem}\left(\dot{\gamma}(t, x), e_{i}(t), \dot{\gamma}(t, x), e_{j}(t)\right)
$$

with initial conditions $J(0, x)=I, \dot{J}(0, x)=\nabla^{2} u(x)$ along the curve $\gamma[u](t, x):=\exp _{x}(t \nabla u(x))$.

ii) if $\nabla^{2} u(x)+\nabla^{2}\left(\frac{1}{2} \rho_{y}^{2}\right)(x) \geq 0$, then, $\operatorname{det} J[u](t, x) \geq 0$ for all $t \in[0,1]$. In particular, det $J[u](t, x)>0$ for all $t \in[0,1)$.

Proof. The proof is contained in Chapter 14 of $[\mathbf{2 0}]$. See the discussion on pp. 365-367 for i) and pp. 412-418 for ii).

Up to now, we have not yet considered the reference measure. Next, we will estimate the Jacobi determinant of $F[u]$ with respect to reference measure $\nu$. For results and their proofs, see Chapter 14 in [20. Jacobi equation (4.1) immediately suggests that behavior of $J[u](t)$ is controlled by curvatures and the Hessian of $u$. However, we are only interested in estimating the Jacobi determinant, which can indeed be controlled by the Ricci curvature. First, we introduce a couple of definitions and notations.

Definition 4.7. Let $(\mathscr{M}, g, \nu)$ be a complete Riemannian metric-measure space and $\Omega \subset \mathcal{M}$ be a domain. Let $u \in C^{2}(\Omega)$. We denote

$$
\mathcal{J}[u](t ; x):=\operatorname{det}(J[u](t, x))
$$

for any $x \in \Omega$, and define

$$
\mathcal{J}_{\nu}[u](t, x):=\lim _{r \rightarrow 0} \frac{\nu\left[F_{t}[u]\left(B_{r}(x)\right)\right]}{\nu\left[B_{r}(x)\right]}=\frac{e^{-V\left(F_{t}[u](x)\right)}}{e^{-V(x)}} \mathcal{J}[u](t, x), \quad \forall x \in \Omega .
$$


We also denote

$$
\mathcal{D}_{N}[u](t, x)= \begin{cases}\left(\mathcal{J}_{\nu}[u](t, x)\right)^{1 / N} & n \leq N<\infty \\ \log \mathcal{J}_{\nu}[u](t, x) & N=\infty\end{cases}
$$
$\mathcal{J}_{\nu}[u]$

The following proposition from Chapter 14 of $[\mathbf{2 0}$ provides an estimate for

Corollary 4.8. Let $u \in C^{2}(\Omega)$. Let $x \in \Omega$ and $\gamma(t, x)$ be a geodesic starting at $x$ with $\dot{\gamma}(t, x)=\nabla u(x)$. Suppose $J[u](t, x)$ is invertible for all $t \in[0,1)$. Then, for any $N \in[n, \infty]$,

$$
\ddot{\mathcal{D}}_{N}[u](t, x) \leq\left\{\begin{array}{ll}
-\frac{1}{N} \operatorname{Ric}_{N, \nu}(\dot{\gamma}(t, x)) \mathcal{D}_{N}[u](t, x) & n \leq N<\infty, \\
-\operatorname{Ric}_{\infty, \nu}(\dot{\gamma}(t, x)) & N=\infty,
\end{array} \quad \forall t \in(0,1)\right.
$$

Proof. See pp. 379-383 in [20].

Remark 4.9. $\mathcal{D}_{\infty}=-l(t)$, where $l(t)$ is defined in $2 \mathbf{2 0}$.

5. Measure Estimate. In this section, we give a proof of Theorem 1.2 . Indeed, all the technical work has been done in the previous two sections.

Proof of Theorem 1.2. To simplify the notation, we write $A=$ $A(E / \Omega, u) \subset \Omega$ in the proof. First, we show that the map $F[u](x)=F[u](1, x)$ is a subjective map from $A$ onto $E$. Fix a point $y \in E$, by definition, there exists a paraboloid $P_{a, y}$ touches $u$ at some $x \in A$. By Lemma 3.6. we know that $P_{a, y}$ is smooth at $x$ and the contact condition implies

$$
\nabla u(x)=-\rho_{y}(x) \nabla \rho_{y}(x) .
$$

Hence, $F[u](1, x)=\exp _{x}\left[-\rho_{y} \nabla \rho_{y}(x)\right]=y$. This proves the subjectivity.

Next, as indicated by Lemma 3.6, $x$ and $y$ are neither cut-points nor conjugate points of each other, Proposition 4.6 together with the contact relation

$$
\nabla^{2} u(x) \geq-\nabla^{2}\left(\frac{1}{2} \rho_{y}^{2}(x)\right)
$$

implies that $J[u](t, x)$ is invertible for all $t \in(0,1)$ and $\mathcal{J}_{\nu}(t, x) \geq 0$ for all $t \in$ $[0,1]$. Therefore, $\mathcal{D}_{N}$ (recall Definition 4.7) satisfies the differential inequality given in Corollary 4.8. Denote $\gamma(t, x)=\exp _{x}(t \nabla u(x))$. By (5.1) and the fact that $\gamma$ is a geodesic, we have

$$
|\dot{\gamma}(t, x)|^{2}=|\dot{\gamma}(0, x)|^{2}=|\nabla u(x)|^{2}, \quad \forall t \in[0,1] .
$$

Combining this with the Ricci lower lower bound condition, the differential inequalities in Corollary 4.8 reduce to

$$
\ddot{\mathcal{D}}_{N}[u](t, x) \leq \begin{cases}-(K / N)|\nabla u(x)|^{2} \mathcal{D}_{N}[u](t, x) & n \leq N<\infty \\ -K|\nabla u(x)|^{2} & N=\infty\end{cases}
$$


Now, applying a standard ODE comparison argument with the initial condition $\mathcal{D}_{N}[u](0, x)=\left\{\begin{array}{ll}1 & n \leq N<\infty \\ 0 & N=\infty\end{array}\right.$ and $\dot{\mathcal{D}}_{N}[u](0, x)= \begin{cases}\frac{1}{N} \Delta_{\nu} u(x) & n \leq N<\infty \\ \Delta_{\nu} u(x) & N=\infty\end{cases}$ we obtain, for all $x \in A$,

$$
\mathcal{D}_{N}[u](1, x) \leq \begin{cases}\mathcal{D}_{K, N}[u](x) & N \in[0, \infty) \\ -\frac{1}{2} K|\nabla u(x)|^{2}+\Delta_{\nu} u(x) & N=\infty .\end{cases}
$$

where $\mathcal{D}_{K, N}[u]$ is given in 1.2 . Finally, since $u$ is $C^{2}$ in $\Omega$ containing $A$ and hence $F[u / a](1, \cdot)$ is differentiable in $\Omega$, we may apply the area formula to obtain

$$
\begin{aligned}
\nu[E] & \leq \int_{A} \mathcal{J}_{\nu}(x) \nu(d x) \\
& \leq \begin{cases}\int_{A}\left[\mathcal{D}_{N}[u](x)\right]^{N} \nu(d x) & n \leq N<\infty, \\
\int_{A} \exp \left[-\frac{1}{2} K|\nabla u(x)|^{2}+\Delta_{\nu} u(x)\right] \nu(d x) & N=\infty .\end{cases}
\end{aligned}
$$

Here we used the fact that $\mathcal{J}_{\nu} \geq 0$. The required formula follows from (5.3).

REMARK 5.1. Just two inequalities were used in the above proof. One in the application of area formula. It becomes equality if and only if $\mathcal{F}[u]$ is oneto-one. The other one is the estimate by the ODE comparison. Differential inequality (5.2) is equivalent to the Ricci lower bounded (see details on p. 400, Proposition 14.8 in $[\mathbf{2 0}]$ ). These two inequalities can simultaneously become equalities.

REMARK 5.2. The formula given by Theorem 1.2 is, in certain sense, a dual formula to the Sobolev inequalities. In particular, the case $N=\infty$ could be viewed as a dual formula for the log-Sobolev inequality. One way to recognize this duality is to consider the key ingredients in the proof of Theorem 1.2 and the proof of Sobolev inequalities. It is known that Sobolev inequalities can be derived as a consequence of co-area formula, while Theorem 1.2 is proved based on the area formula.

In the rest of this paper, we will not explore the full power of the above estimate. The following corollary will suffice to prove Harnack inequalities.

Corollary 5.3. Let $(\mathscr{M}, g, \nu)$ be a complete Riemannian metric-measure space of dimension $n \geq 2$. Let $E$ be a closed subset of a geodesic ball $B_{r}$ and $u \in C\left(\bar{B}_{r}\right)$. Let $K \geq 0$ and $N \in[n, \infty)$ be two constants.

Suppose $a>0$ and $A\left(a, E / B_{r}, u\right) \subset B_{r}$, and assume that there exists a subdomain $\Omega^{\prime}$ containing $A\left(a, E / B_{r}, u\right)$ such that $u \in C^{2}\left(\Omega^{\prime}\right)$. Then we have

$$
\left.\operatorname{Ric}_{N, \nu}\right|_{B_{r}} \geq-K g \Rightarrow \nu[E] \leq \int_{A\left(a, E / B_{r}, u\right)}\left\{\mathcal{D}_{K, N, r}[u / a](x)\right\}^{N} \nu(d x),
$$


where for any $x \in A(a, E / \Omega, u)$,

$$
\mathcal{D}_{K, N, r}[u / a](x):=\frac{\sinh (r \omega)}{r \omega}\left[r \omega \operatorname{coth}(r \omega)+\frac{\Delta_{\nu} u(x)}{N a}\right],
$$

where $\omega=2 \sqrt{\frac{K}{N}}$ is defined in 2.3.). Moreover, in the case $K=0$, expressions are understood as their obvious limits.

Proof. Apply the proof of Theorem 1.2 to the function $\frac{1}{a} u(x)$ and observe that at a contact point $x \in A\left(a, E / B_{r}, u\right)$, we have

$$
\frac{1}{a} \nabla u(x)=-\rho_{y}(x) \nabla \rho_{y}(x) .
$$

It follows from $A\left(a, E / B_{r}, u\right) \cup E \subset B_{r}$ that

$$
\frac{1}{a}|\nabla u(x)|=\left|\rho_{y}(x)\right| \leq 2 r, \forall x \in A .
$$

The proof is then completed by substituting this estimate to the ODE estimate.

6. Ricci Comparison and A Barrier. In this section, we recall the Ricci comparison theorem for the modified Laplacian operator and use it to construct a barrier function which will be used later (proof of Lemma 9.3). Recall the following result from $[\mathbf{1 7}$ (or $[\mathbf{1 3},[\mathbf{2 3}]$ ).

Proposition 6.1. Suppose $\left.\operatorname{Ric}_{N, \nu}\right|_{B_{R}} \geq-K g, K \geq 0, N \in[0, \infty)$. Then for any two points $x, y \in B_{R}$, we have

$$
\Delta_{\nu} \rho_{y}(x) \leq(N-1) \frac{\mathcal{H}\left(2 \sqrt{\frac{K}{N-1}} \rho_{y}(x)\right)}{\rho_{y}(x)}
$$

in the support sense everywhere (recall notation 2.4 from Section 2).

REMARK 6.2. Note that it is easy to check

$$
1+(N-1) \mathcal{H}\left(2 \sqrt{\frac{K}{N-1}} \rho\right) \leq N \mathcal{H}\left(2 \sqrt{\frac{K}{N}} \rho\right) .
$$

Therefore, we have

$$
\Delta_{\nu} \frac{\rho_{y}^{2}}{2}(x) \leq N \mathcal{H}\left(\omega \rho_{y}(x)\right) .
$$

The rest of this section is devoted to the construction of a barrier function. A similar construction was given in [2] (also adopted in [10]). However, as working with potentially negative curvature, one needs more detailed information on such a barrier function to ensure that the constants depend on curvatures in a proper way (in particular, this is necessary in studying elliptic 
fully-nonlinear PDEs). This information is provided in the following lemmas. Their proofs are technical but completely routine. Recall the definition of the constant $\alpha$ from Section 2, Note that $\alpha \geq 2$.

Lemma 6.3. There exists a function $h:[0, \infty) \rightarrow \mathbb{R}$ such that

i) $h \in C^{2}[0, \infty)$ and $h^{\prime}(0)=0$;

ii) $\inf _{[0, \infty)} h \geq-\alpha^{2}(18)^{\alpha}$;

iii) The derivatives of $h$ satisfy the following estimates:

(a) for $t>1 / 18$,

$$
h^{\prime \prime}(t)-\frac{h^{\prime}(t)}{t}=-\alpha(\alpha+2) t^{-(\alpha+2)}<0, \quad \frac{h^{\prime}(t)}{t}=\alpha t^{-(\alpha+2)}>0 ;
$$

(b) for $0 \leq t \leq 1 / 18$,

$$
\left|h^{\prime \prime}(t)-\frac{h^{\prime}(t)}{t}\right| \leq 2 \alpha^{2}(18)^{\alpha+3}, \quad 0<\frac{h^{\prime}(t)}{t} \leq 500 \alpha^{2}(18)^{\alpha} .
$$

Proof. Let $\beta_{i}, i=0,1,2$ be constants to be determined. Consider the function

$$
h(t):= \begin{cases}\beta_{0}+\beta_{1} t^{2}+\beta_{2} t^{4} & t \leq \frac{1}{18} \\ (1 / 18)^{-\alpha}-t^{-\alpha} & t>\frac{1}{18} .\end{cases}
$$

By choosing

$$
\begin{aligned}
& \beta_{0}=-2^{(-3+\alpha)} 9^{\alpha}\left(6 \alpha+\alpha^{2}\right), \\
& \beta_{1}=2^{\alpha} 9^{(2+\alpha)}\left(4 \alpha+\alpha^{2}\right), \\
& \beta_{2}=-2^{(1+\alpha)} 9^{(4+\alpha)}\left(2 \alpha+\alpha^{2}\right),
\end{aligned}
$$

we match up the values of the first two derivatives of $h$ at $t=1 / 18$. It is easy to check $h$ has all the required properties.

Lemma 6.4. Let $(\mathscr{M}, g, \nu)$ be a complete Riemannian metric-measure space. Fix a geodesic ball $B_{r}\left(x_{0}\right)$ with $r \leq R$. Let $K \geq 0$ and $1<N<\infty$. Suppose that $\left.\operatorname{Ric}_{N, \nu}\right|_{B_{r}\left(x_{0}\right)} \geq-K g$. Then there exists a function $\psi$ such that

i) $\psi$ is continuous in $B_{r}\left(x_{0}\right)$ and lies in $C^{2}\left(B_{r}\left(x_{0}\right) \backslash C u t\left(x_{0}\right)\right)$;

ii) $\inf _{\left.B_{r}\left(x_{0}\right)\right)} \psi \geq-\alpha^{2}(18)^{\alpha}$ and

$\psi \geq(18)^{\alpha}-(4 / 3)^{\alpha}$ in $B_{r}\left(x_{0}\right) \backslash B_{3 r / 4}\left(x_{0}\right), \quad \psi=(18)^{\alpha}-2^{\alpha}$ on $\partial B_{r / 2}\left(x_{0}\right)$;

iii) $\psi$ is locally bounded above in support sense in $B_{r}\left(x_{0}\right)$;

iv) in $\bar{B}_{r / 18}\left(x_{0}\right) \backslash \operatorname{Cut}\left(x_{0}\right)$,

$$
\frac{r^{2} \Delta_{\nu} \psi}{N}+\mathcal{H}(\omega r) \leq 2 \alpha^{3}(18)^{\alpha+3}
$$


v) in $B_{r}\left(x_{0}\right) \backslash\left(\bar{B}_{r / 18}\left(x_{0}\right) \cup C u t\left(x_{0}\right)\right)$

$$
\frac{r^{2} \Delta_{\nu} \psi}{N}+\mathcal{H}(\omega r) \leq 0
$$

Here, the constants $\omega$ and $\alpha$ are defined as in Section 2 .

Proof. Take $h(t)$ to be the function given in Lemma 6.3 with $\alpha$ as given in 2.6. Denote $\rho_{x_{0}}$ by $\rho$ for convenience. Define

$$
\psi:=h(\rho / r) .
$$

It is routine to check that $\psi$ has all the required properties by Lemma 6.3 and Proposition 6.1.

7. Measure Doubling and Monotonicity of $\mathcal{I}_{K, N}$. In this section, we summarize some results about the measure doubling property, the integration $\mathcal{I}\left(f, B_{r}, q\right)$ (defined in Section 2 ) and some basic $L^{p}$ theory which will be used in the proof of Harnack inequalities (Theorems 1.3 1.5). First, we recall the doubling property (see Definition 18.1 in [20]).

Definition 7.1. Let $(\mathcal{M}, g, \nu)$ be a metric measure space. The measure $\nu$ is said to be doubling if there exists a constant $\mathscr{D}$ such that

$$
\nu\left[B_{2 r}(x)\right] \leq \mathscr{D} \nu\left[B_{r}(x)\right] \quad \text { for any } x \in \mathcal{M} \text { and } r>0 .
$$

The measure $\nu$ is said to be locally doubling if for any fixed closed ball $B_{R}(x) \subset$ $\mathcal{M}$, there is a constant $\mathscr{D}=\mathscr{D}(x, R)$ such that

$$
\nu\left[B_{2 r}(x)\right] \leq \mathscr{D} \nu\left[B_{r}(x)\right] \quad \text { for any } x \in B_{R}(x) \text { and } r \in(0, R) .
$$

The following proposition provides estimates for the doubling constant on a Riemannian metric-measure space in terms of Ricci lower bound (Corollary 18.11 in 20$]$ ).

Proposition 7.2. Let $(\mathscr{M}, g, \nu)$ be a Riemannian metric-measure space satisfying the curvature condition $\operatorname{Ric}_{N, \nu} \geq-K g$ for some $K \geq 0$ and $1<$ $N<\infty$. Then $\nu$ is doubling with a constant which is:

- uniform and less or equal to $2^{N}$ if $K=0$;

- locally uniform and less or equal to $2^{N}\left[\cosh \left(2 \sqrt{\frac{K}{N-1}} R\right)\right]^{N-1}$ for any $B_{R}$, if $K>0$.

Recall the definition of the constants $\mathscr{D}_{r}$ and $\eta_{r}$ given by (2.3) in Section 2. We see that

$$
2^{N}\left[\cosh \left(2 \sqrt{\frac{K}{N-1}} R\right)\right]^{N-1} \leq \mathscr{D}_{R}
$$


Moreover, the doubling property allows the following simple estimate

$$
\frac{\nu\left[B_{r_{1}}(x)\right]}{\nu\left[B_{r_{2}}(x)\right]} \leq \mathscr{D}_{R} \cdot\left(\frac{r_{1}}{r_{2}}\right)^{N \eta_{R}}
$$

providing $B_{r_{2}}(x) \subset B_{r_{1}}(x) \subset B_{R}$ on $(\mathscr{M}, g, \nu)$ with $\left.\operatorname{Ric}_{N, \nu}\right|_{B_{R}} \geq-K g$.

Recall the definition of $\mathcal{I}\left(f ; B_{r}, q\right)$ (2.5) from Section 2. The integral $\mathcal{I}\left(f ; B_{r}, q\right)$ has good monotonicity property and fits the scaling well.

Lemma 7.3. For any $1<N<\infty$, we have

i) $\mathcal{I}\left(f ; B_{r}, t\right) \leq \mathcal{I}\left(f ; B_{r}, s\right)$ whenever $t<s$.

ii) If $B_{r_{1}}(x) \subset B_{r_{2}}(x) \subset B_{R}$, then $\mathcal{I}\left(f ; B_{r_{1}}(x), \eta_{R}\right) \leq \mathcal{I}\left(f ; B_{r_{2}}(x), \eta_{R}\right)$.

Proof. i) follows from the standard $L^{p}$ theory; ii) follows from direct calculation and (7.1).

Given a function $f$ on the domain $\Omega$ with finite measure, we define

$$
\tilde{\lambda}_{\Omega}(t):=\frac{\nu[\{f \geq t\} \cap \Omega]}{\nu[\Omega]} .
$$

When no confusion could arise, we shall omit the subscript. We shall need the following well-known statement in $L^{p}$-theory (see $[3]$ for instance).

Lemma 7.4. Let $C>1$. Then, for any $0<p<\infty$,

$$
f_{\Omega} f^{p}<\infty \Leftrightarrow S:=\sum_{k=0}^{\infty} C^{p k} \tilde{\lambda}\left(C^{k}\right)<\infty
$$

and

$$
\left(1-\frac{1}{C^{p}}\right) S+\frac{1}{C^{p}} \tilde{\lambda}(1) \leq f_{\Omega} f^{p} \leq 1+\left(C^{p}-1\right) S .
$$

8. Proof of Harnack Inequalities I. In this section, we establish the key lemma for the proof of Harnack inequalities (Theorems 1.3 1.5). It describes the local growth of the solution $u$. A similar lemma was used in [2]. Our proof is essentially the same as that in $[\mathbf{2}$ (also in $[\mathbf{1 0}]$ ). However, by using some fine properties of the contact sets, we avoid the approximation procedures required in $\overline{\mathbf{2}}$ and $\overline{\mathbf{1 0}}$. Recall from Section 2 the constants $M, \mu, \delta_{0}$ (2.6, 2.8), the function $\mathcal{H}(t), \mathcal{S}(t)(2.4)$ in Section 2 and integral $\mathcal{I}\left(f ; B_{r}, q\right)$ (2.5).

LEMMA 8.1. Let $(\mathscr{M}, g, \nu)$ be a complete metric-measure space. Let $u \in$ $C\left(\bar{B}_{2 R}\right) \cap C^{2}\left(B_{2 R}\right)$ and $f \in C\left(B_{2 R}\right)$. Let $K \geq 0, N<\infty$. Suppose that

$$
\left.\operatorname{Ric}_{N, \nu}\right|_{B_{2 R}} \geq-K g, \quad \mathcal{I}\left(f, B_{2 R}, 1\right) \leq \delta_{0} .
$$


Then, for any given ball $B_{2 r}\left(x_{0}\right) \subset B_{2 R}$,

$$
u \geq 0 \text { in } B_{r}\left(x_{0}\right), \quad \inf _{B_{r / 2}\left(x_{0}\right)} u \leq 1, \quad \Delta_{\nu} u \leq f \text { in } B_{r}\left(x_{0}\right),
$$

implies

$$
\frac{\nu\left[\{u \leq M\} \bigcap B_{r / 18}\left(x_{0}\right)\right]}{\nu\left[B_{r}\left(x_{0}\right)\right]} \geq \mu,
$$

where $M$ and $\mu$ are uniform constants defined by (2.6) in Section 2 .

Proof. Let $\psi$ be the function constructed in Lemma 6.4 with respect to $B_{r}\left(x_{0}\right)$. Consider $w=u+\psi$ on $B_{r}\left(x_{0}\right)$. By the construction of $\psi$ and the assumptions on $u$, there exists $y_{0} \in B_{r / 2}\left(x_{0}\right)$ such that

$$
w\left(y_{0}\right)=\inf _{B_{r / 2}\left(x_{0}\right)} w \leq 1+(18)^{\alpha}-2^{\alpha},
$$

and $w$ satisfies

$$
\inf _{B_{r}\left(x_{0}\right) \backslash B_{3 r / 4}\left(x_{0}\right)} w \geq(18)^{\alpha}-(4 / 3)^{\alpha} .
$$

These two conditions together with Lemma 3.7 and $\alpha \geq N \geq 2$ 2.7 imply

$$
A\left(\frac{1}{r^{2}}, \bar{B}_{r / 6}\left(y_{0}\right) / B_{r}\left(x_{0}\right), w\right) \subset \subset B_{r}\left(x_{0}\right) \bigcap\left\{w \leq 1+(18)^{\alpha}-2^{\alpha}+\frac{1}{36}\right\} .
$$

To simplify the notation in the rest of the proof, we denote

$$
A:=A\left(\frac{1}{r^{2}}, \bar{B}_{r / 6}\left(y_{0}\right) / B_{r}\left(x_{0}\right), w\right),
$$

By ii) of Lemma 6.4, we obtain

$$
w=u+\psi \leq \frac{37}{36}+(18)^{\alpha}-2^{\alpha} \Rightarrow u \leq 2 \alpha^{2}(18)^{\alpha} .
$$

This along with 8.2 and the definition of $M(2.6)$ implies

$$
\nu\left[A \cap B_{r / 18}\left(x_{0}\right)\right] \leq \nu\left[\{u<M\} \cap B_{r / 18}\left(x_{0}\right)\right] .
$$

Hence, it suffices to estimate $\nu\left[A \cap B_{r / 18}\left(x_{0}\right)\right]$ from below.

By Lemma 6.4, $\psi$ is locally bounded above in support sense. Since $u$ is $C^{2}$, $w$ is locally bounded from both above and below on $A$ in the support sense. This implies

$$
A \cap \operatorname{Cut}\left(x_{0}\right)=\emptyset
$$

because $\psi$, and hence $w$, are not bounded below in support sense on $C u t\left(x_{0}\right)$. We also note that $A$ is closed (Lemma 3.3) and $C u t\left(x_{0}\right)$ is also closed. Therefore, there is a neighborhood $\Omega^{\prime} \subset B_{r / 4}\left(y_{0}\right)$ of $A$ such that $w \in C^{2}\left(\Omega^{\prime}\right)$. Then, we apply Corollary 5.3 to obtain

$$
\nu\left[B_{r / 6}\left(y_{0}\right)\right] \leq \int_{A}\left\{\mathcal{D}_{K, N, r}\left[r^{2} w\right](x)\right\}^{N} \nu(d x)
$$


with $\mathcal{D}_{K, N, r}\left[r^{2} w\right](x)=\mathcal{S}(\omega r)\left[\mathcal{H}(\omega r)+\frac{r^{2} \Delta_{\nu} w(x)}{N}\right]$.

Next, we estimate $\mathcal{D}_{K, N, r}\left[r^{2} w\right]$. First, Lemma 6.4 together with $\Delta_{\nu} u \leq f$ implies that, for any $x \in A \cap \bar{B}_{r / 18}\left(x_{0}\right)$,

$$
\mathcal{D}_{K, N, r}\left[r^{2} w\right](x) \leq 2 \alpha^{3}(18)^{\alpha+3} \mathcal{S}(\omega r)+\mathcal{S}(\omega r)\left(\frac{r^{2} f^{+}(x)}{N}\right) ;
$$

and for any $x \in A \cap\left(B_{r}\left(x_{0}\right) \backslash \bar{B}_{r / 18}\left(x_{0}\right)\right)$,

$$
\mathcal{D}_{K, N, r}\left[r^{2} w\right](x) \leq \mathcal{S}(\omega r)\left(\frac{r^{2} f^{+}(x)}{N}\right) .
$$

Here, $f^{+}=\max \{f, 0\}$. These estimates along with the simple relation

$$
(t+s)^{N}=\left((t+s)^{+}\right)^{N} \leq 2^{N-1}\left[\left(t^{+}\right)^{N}+\left(s^{+}\right)^{N}\right] \text { for } t+s>0,
$$

imply

$$
\begin{aligned}
\int_{A}\left\{\mathcal{D}_{K, N, r}\left[r^{2} w\right](x)\right\}^{N} \nu(d x) \leq & \frac{1}{2}\left[4 \alpha^{2}(18)^{\alpha+3} N \cosh (\omega R)\right]^{N} \nu\left[A \cap B_{r / 18}\left(x_{0}\right)\right] \\
& +2^{N-1} \mathcal{S}^{N}(\omega r) \int_{B_{r}\left(x_{0}\right)}\left(\frac{r^{2} f^{+}(x)}{N}\right)^{N} .
\end{aligned}
$$

Combining this estimate with (8.4), we obtain

$$
\begin{aligned}
1 \leq & \frac{1}{2}\left[4 \alpha^{2}(18)^{\alpha+3} N \cosh (\omega R)\right]^{N} \nu\left[A \cap B_{r / 18}\left(x_{0}\right)\right] / \nu\left[B_{r / 6}\left(y_{0}\right)\right] \\
& +2^{N-1} \mathcal{S}^{N}(\omega r) \frac{1}{\nu\left[B_{r / 6}\left(y_{0}\right)\right]} \int_{B_{r}\left(x_{0}\right)}\left(\frac{r^{2} f^{+}(x)}{N}\right)^{N} .
\end{aligned}
$$

By the doubling property, we have

$$
\nu\left[B_{r}\left(x_{0}\right)\right] \leq \nu\left[B_{3 r / 2}\left(y_{0}\right)\right] \leq \mathscr{D}_{2 R}^{4} \nu\left[B_{r / 6}\left(x_{0}\right)\right] .
$$

Applying monotonicity of the integral $\mathcal{I}$ (Lemma 7.3), we obtain

$$
\frac{1}{\nu\left[B_{r / 6}\left(y_{0}\right)\right]} \int_{B_{r}\left(x_{0}\right)}\left(\frac{r^{2} f^{+}(x)}{N}\right)^{N} \leq \mathscr{D}_{2 R}^{4} f_{B_{r}\left(x_{0}\right)}\left(\frac{r^{2} f^{+}(x)}{N}\right)^{N} \leq \mathscr{D}_{2 R}^{4} \delta_{0}^{N}
$$

Combining (8.8) with (8.10) and recalling the choice of $\delta_{0}$ (2.8), we obtain

$$
\left.4 \alpha^{2}(18)^{\alpha+3} N \cosh (2 \omega R)\right]^{N} \mathscr{D}_{2 R}^{4} \frac{\nu\left[A \cap B_{r / 18}\left(x_{0}\right)\right]}{\nu\left[B_{r}\left(x_{0}\right)\right]} \geq 1 .
$$


Recalling the definition of $\mu$ (2.6), we then have

$$
\frac{\nu\left[A \cap B_{r / 18}\left(x_{0}\right)\right]}{\nu\left[B_{r}\left(x_{0}\right)\right]} \geq\left[4 \alpha^{2}(18)^{\alpha+3} N \cosh (\omega R)\right]^{-N} \mathscr{D}_{2 R}^{-4} \geq \mu .
$$

The proof is completed by combining the above inequality with (8.3).

9. Proof of Harnack Inequalities II. In this section, we give a proof of Theorem 1.3. The idea here is essentially the same as in [2], which goes back to $[\mathbf{1 1}$ and $[\mathbf{1 2}]$. Our presentation here follows $[\mathbf{1 8}$. First, we recall the following version of Vitali's covering lemma. One may refer to a standard textbook in measure theory for a proof.

Lemma 9.1. Let $(X, \rho, \nu)$ be a metric-measure space. Let $\mathcal{V}$ be a family of closed balls of nonzero radius in $X$ and $D$ be the collection of centers of these balls. Suppose that

$$
\sup \{\operatorname{diam}(B): B \in \mathcal{V}\}<\infty
$$

and $\nu$ satisfies the local measure doubling property, that is, for any compact set $Z, \nu$ has a doubling constant depending on $Z$. Then there exists a countable sub-collection $\mathcal{V}^{\prime}$ of $\mathcal{V}$ such that

$$
D \subset \bigcup_{B \in \mathcal{V}^{\prime}} B
$$

and the collection $\left\{\frac{1}{4} B: B \in \mathcal{V}^{\prime}\right\}$ is disjoint.

Recall the definitions of the constants $M, \mu, \delta_{0}$ and the integral $\mathcal{I}\left(f ; B_{r}, q\right)$ from Section 2. Theorem 1.3 follows immediately from the following two lemmas.

Lemma 9.2. Under the assumptions of Theorem 1.3, denote by $D_{k}$ the set

$$
D_{k}:=\left\{x \in B_{R / 2}: u(x) \leq M^{k}\right\} .
$$

Suppose additionally that

$$
\inf _{B_{R / 2}} u \leq 1 \text { and } \mathcal{I}\left(f, B_{2 R}, \eta_{2 R}\right) \leq \delta_{0} .
$$

Then, for any $k \geq 0$

$$
\nu\left[D_{k+1} \cap B_{r_{x} / 4}(x)\right] \geq \mu \nu\left[B_{r_{x}}(x)\right]
$$

for all $x \in B_{R / 2} \backslash D_{k}$ and $r_{x}=\operatorname{dist}\left(x, D_{k}\right)$.

Proof. Fix $x_{0} \in B_{R / 2} \backslash D_{k}$. In the rest of the proof, we write $r_{0}=r_{x_{0}}=$ $\operatorname{dist}\left(x_{0}, D_{k}\right)$ for convenience. Since $D_{0} \neq \emptyset$, we have $r_{0} \leq R / 2$. Let $z_{0}$ denote the center of $B_{2 R}$. Connect $x_{0}$ and $z_{0}$ by a minimizing geodesic. Choose $y_{0}$ be a point on this geodesic such that $\rho\left(y_{0}, x_{0}\right)=r_{0} / 8$ and consider the ball $B_{r_{0} / 8}\left(y_{0}\right)$. 
As $\gamma$ is minimizing, we have $\rho\left(y_{0}, z_{0}\right)+\rho\left(y_{0}, x_{0}\right)=\rho\left(z_{0}, x_{0}\right)$. Then, by triangle inequality and the estimate $r_{0} \leq R / 2$, we have

$$
B_{r_{0} / 8}\left(y_{0}\right) \subset B_{r_{0} / 4}\left(x_{0}\right) \cap B_{R / 2} .
$$

Therefore

$$
B_{r_{0} / 4}\left(x_{0}\right) \cap D_{k+1} \supset B_{r_{0} / 8}\left(y_{0}\right) \cap\{w \leq M\},
$$

where $w=u / M^{k}$. Thus, it suffices to estimate $\nu\left[B_{r_{0} / 8}\left(y_{0}\right) \cap\{w \leq M\}\right]$ from below.

Consider the ball $B_{l}\left(y_{0}\right)$ with $l=\frac{9}{4} r_{0}$. First, as $r_{0}<R / 2$, we have $l \leq \frac{9}{8} R$ and by triangle inequality $B_{2 l}\left(y_{0}\right) \subset B_{2 R}$. Second, since

$$
\operatorname{dist}\left(y_{0}, D_{k}\right) \leq \rho\left(x_{0}, y_{0}\right)+\operatorname{dist}\left(x_{0}, D_{k}\right) \leq \frac{9}{8} r_{0},
$$

we have $\bar{B}_{l / 2} \cap D_{k} \neq \emptyset$. Third, noticing that $\frac{l}{18}=\frac{r_{0}}{8}$, we obtain

$$
B_{l / 18}\left(y_{0}\right) \cap\{w \leq M\}=B_{r_{0} / 8}\left(y_{0}\right) \cap\{w \leq M\} .
$$

With these three elementary relations, we may apply the Lemma 8.1 to $w$ on $B_{l}\left(y_{0}\right)$ and obtain

$$
\nu\left[B_{r_{0} / 8} \cap\{w \leq M\}\right] \geq \mu \nu\left[B_{l}\left(y_{0}\right)\right] \geq \mu \nu\left[B_{r_{0}}\left(y_{0}\right)\right] .
$$

Here the last inequality follows from the fact that

$$
B_{l}\left(y_{0}\right)=B_{9 r_{0} / 4}\left(y_{0}\right) \supset B_{r_{0}}\left(x_{0}\right) .
$$

Combining (9.1) and (9.2), we complete the proof.

LEMMA 9.3. Under the assumptions of Theorem 1.3, and assuming additionally that

$$
\inf _{B_{R / 2}} u \leq 1 \text { and } \mathcal{I}\left(f ; B_{2 R} ; \eta_{2 R}\right) \leq \delta_{0},
$$

we have, for any $k \geq 1$,

$$
\tilde{\lambda}_{B_{R / 2}}\left(M^{k}\right) \leq(1-\mu)^{k}
$$

where $\tilde{\lambda}_{B_{R / 2}}\left(M^{k}\right)$ is the distribution function of $u$ in $B_{R / 2}$ defined by 7.2).

Proof. Recall $D_{k}:=\left\{x \in B_{R / 2}: u(x) \leq M^{k}\right\}$. Claim: for any $k \geq 1$

$$
\nu\left[\left(D_{k+1} \backslash D_{k}\right) \cap B_{R / 2}\right] \geq \mu \nu\left[B_{R / 2} \backslash D_{k}\right] .
$$

Consider the cover $\mathcal{V}$ of the set $B_{R / 2} \backslash D_{k}$ defined by

$$
\mathcal{V}:=\left\{\bar{B}_{r_{x}}(x): x \in B_{R / 2} \backslash D_{k}, r_{x}=d\left(x, D_{k}\right)\right\} .
$$

By Lemma 9.2, we have

$$
\nu\left[D_{k+1} \cap B_{r_{x} / 4}(x)\right] \geq \mu \nu\left[B_{r_{x}}(x)\right] .
$$


Using Vitali's covering lemma, we may take a sequence of balls $B_{r_{i}}\left(x_{i}\right) \in \mathcal{V}$ such that $B_{r_{i} / 4}\left(x_{i}\right)$ are pairwise disjoint and $B_{R / 2} \backslash D_{k} \subset \cup_{i} B_{r_{i}}\left(x_{i}\right)$. Moreover, by the choice of $r_{x}$, we also have $B_{r_{i} / 4}\left(x_{i}\right) \cap D_{k}=\emptyset$ and henceforth,

$$
\cup_{i} B_{r_{i} / 4} \cap B_{R / 2} \subset B_{R / 2} \backslash D_{k} .
$$

Now, we compute

$$
\begin{aligned}
\nu\left[B_{R / 2} \backslash D_{k}\right] & \leq \nu\left[\cup_{i} B_{r_{i}}\left(x_{i}\right)\right] \leq \sum_{i} \nu\left[B_{r_{i}}\left(x_{i}\right)\right] \leq \sum_{i} \frac{1}{\mu} \nu\left[D_{k+1} \cap B_{r_{i} / 4}\left(x_{i}\right)\right] \\
& =\frac{1}{\mu} \nu\left[\cup_{i}\left(D_{k+1} \cap B_{r_{i} / 4}\left(x_{i}\right)\right)\right]=\frac{1}{\mu} \nu\left[D_{k+1} \cap\left(\cup_{i} B_{r_{i} / 4}\left(x_{i}\right)\right)\right] \\
& \leq \frac{1}{\mu} \nu\left[D_{k+1} \cap\left(B_{R / 2} \backslash D_{k}\right)\right] .
\end{aligned}
$$

Here, in the first equality in the second line, we have used the fact that the balls $\left\{B_{r_{i} / 4}\right\}_{i}$ are disjoint. This completes the proof of the claim.

Again, recalling the definition of $\tilde{\lambda}(t)=\tilde{\lambda}_{B_{R / 2}}(t) \sqrt{7.2}$, it is a direct consequence of the above claim that

$$
\tilde{\lambda}\left(M^{k+1}\right) \leq(1-\mu) \tilde{\lambda}\left(M^{k}\right) .
$$

The required estimate follows by applying this inequality inductively.

Proof of Theorem 1.3. By rescaling $u$ as

$$
\tilde{u}=\frac{u}{e^{1 / p_{0}}\left[\inf _{B_{R / 2}} u+\mathcal{I}\left(f, B_{2 R}, \eta_{2 R}\right)\right]},
$$

we have

$$
\Delta \tilde{u} \leq e^{-1 / p_{0}}\left[\inf _{B_{R / 2}} u+\mathcal{I}\left(f ; B_{2 R} ; \eta_{2 R}\right)\right]^{-1} f:=\tilde{f} .
$$

Then, it follows from ii) in Lemma 2.5 that

$$
\inf _{B_{R / 2}} \tilde{u} \leq 1, \text { and } \mathcal{I}\left(\tilde{f}, B_{2 R}, \eta_{2 R}\right) \leq \frac{1}{e^{1 / p_{0}}} \leq \delta_{0}
$$

On the other hand, from i) in Lemma 2.5, we know that $p_{0}$ satisfies

$$
1+\left(M^{p_{0}}-1\right) \sum_{k=0}^{\infty} M^{p_{0} k} \tilde{\lambda}\left(M^{k}\right) \leq e .
$$

Applying Lemma 9.3 and Lemma 7.4 together with the above inequality, we have

$$
\left(f_{B_{R / 2}} \tilde{u}^{p_{0}}\right)^{1 / p_{0}} \leq e^{1 / p_{0}} .
$$

The required estimate is then obtained by re-normalizing $\tilde{u}$ back. 
10. Proof of Harnack Inequalities III. In this section, we give proofs of Theorem 1.4 and Theorem 1.5. Recall the constant $p_{0}$ in the statement of Theorem 1.3 (see Lemma 2.5), and the constants $\delta_{0}, M, \mu, p_{1}$ from Section 2. Again, they are chosen w.r.t to the large ball $B_{2 R}$. The key role in our proof of the Harnack inequalities plays the following lemma.

LEMma 10.1. Under the assumption of Theorem 1.4, and assuming additionally that

$$
\left(f_{B_{R}}\left(u^{+}\right)^{p_{0}}\right)^{1 / p_{0}} \leq 1, \quad \mathcal{I}\left(f, B_{2 R}, \eta_{2 R}\right) \leq \delta_{0}
$$

and $\beta:=u\left(x_{0}\right)>M, B_{r_{0}}\left(x_{0}\right) \subset B_{R}$ with $r_{0}=R C_{3} \beta^{-p_{1}}$. Then

$$
\sup _{B_{r_{0}}\left(x_{0}\right)} u \geq \beta(1+1 / M) \text {. }
$$

Proof. We argue by contradiction. Suppose that

$$
\sup _{B_{r_{0}}\left(x_{0}\right)} u<\beta(1+1 / M) \text {. }
$$

Consider the function

$$
w=\frac{\beta(1+1 / M)-u}{\beta / M} \text { on } B_{r^{\prime}}\left(y_{0}\right)
$$

with $r^{\prime}=r_{0} / 4$ and $\rho\left(y_{0}, x_{0}\right)=r_{0} / 8$. Note that $w \geq 0$ satisfies

$$
\Delta_{\nu} w \leq \frac{-f}{\beta / M} \leq|f| \text { in } B_{r^{\prime}}\left(x_{0}\right)
$$

and

$$
\inf _{B_{r^{\prime} / 2}\left(y_{0}\right)} w \leq w\left(x_{0}\right) \leq 1 .
$$

Thus, we may apply the Lemma 8.1 to obtain

$$
\mu \nu\left[B_{r^{\prime}}\left(y_{0}\right)\right] \leq \nu\left[\{w \leq M\} \cap B_{r^{\prime} / 18}\left(y_{0}\right)\right] .
$$

Observe that $w \leq M$ implies $u \geq \beta / M$. Therefore, (10.1) together with the Chebyshev's inequality implies that

$$
\beta \leq \frac{M}{\mu^{1 / p_{0}}}\left(f_{B_{r^{\prime}}\left(y_{0}\right)}\left(u^{+}\right)^{p_{0}}\right)^{1 / p_{0}} \leq \frac{M}{\mu^{1 / p_{0}}}\left(\frac{\nu\left[B_{R}\right]}{\nu\left[B_{r^{\prime}}\left(y_{0}\right)\right]}\right)^{1 / p_{0}}
$$

Now, recalling the choice of $r^{\prime}=r_{0} / 4$ and $p_{1}(2.8)$, and applying doubling estimate (7.1) to $B_{r^{\prime}}\left(y_{0}\right) \subset B_{R}\left(y_{0}\right) \subset B_{2 R}$, we obtain

$$
\beta \leq \frac{M}{\mu^{1 / p_{0}}} \mathscr{D}_{2 R}\left(\frac{R}{r^{\prime}}\right)^{N \eta_{2 R} / p_{0}} \leq \frac{M}{\mu^{1 / p_{0}}} \mathscr{D}_{2 R}\left(\frac{4}{C_{3}}\right)^{N \eta_{2 R} / p_{0}} \beta .
$$


By the choice of $C_{3} 2.8$ and Lemma 2.4 the above inequality implies $\beta<\beta$, which is impossible.

Finally, both Theorem 1.4 and Theorem 1.5 can be deduced from Lemma 10.1. Recall the constants $M, p_{1}, C_{3}, \mu$ from Section 2 .

Proof of Theorem 1.4. We first prove Theorem 1.4 with $p=p_{0}$. As in the proof of Theorem 1.3 , by rescaling $u$ as

$$
\tilde{u}=\left[\left(f_{B_{R}}\left(u^{+}\right)^{p_{0}}\right)^{1 / p_{0}}+\mathcal{I}\left(f ; B_{2 R}, \eta_{2 R}\right) / \delta_{0}\right]^{-1} u,
$$

we may assume that $u$ satisfies the hypothesis of Lemma 10.1. Thus, it suffices to bound $\sup u$ by the constant $C_{1}\left(p_{0}\right)$ given in (2.9). Again, we argue by contradiction. Suppose that there exists $x_{0} \in B_{R / 2}$ such that

$$
\beta:=u\left(x_{0}\right)>C_{1}\left(p_{0}\right) .
$$

Consider the sequence $\left\{x_{k}\right\}$ defined as follows: choose inductively $x_{k+1}$ so that

$$
u\left(x_{k+1}\right)=\sup _{B_{r_{k}}\left(x_{k}\right)} u, \quad r_{k}=R C_{3} u\left(x_{k}\right)^{-p_{1}} .
$$

The hypothesis on $\beta>C_{1}\left(p_{0}\right)$ and the choice of $C_{1}\left(p_{0}\right)$ together imply

$$
R C_{3} \sum_{k=0}^{\infty} \frac{1}{\beta^{p_{1}}(1+1 / M)^{k p_{1}}} \leq R / 3
$$

Hence, we may inductively apply Lemma 10.1 to obtain

$$
u\left(x_{k}\right) \geq C_{1}\left(p_{0}\right)(1+1 / M)^{k}, r_{k}<\frac{R C_{3}}{C_{1}^{p_{1}}\left(p_{0}\right)(1+1 / M)^{p_{1} k}}, \sum_{k} r_{k} \leq \frac{R}{3} .
$$

However, this implies $x_{k} \in B_{r_{k}}\left(x_{k}\right) \subset B_{R}$ for all $k \geq 0$ and $u\left(x_{k}\right)$ tends to $\infty$ in $B_{R}$. This contradicts the fact that $u$ is continuous in $\bar{B}_{R}$ and hence proves the case $p=p_{0}$.

For $p>p_{0}$, one may take $C_{1}(p)=C_{1}\left(p_{0}\right)$ and the required inequality follows from standard $L^{p}$ theory. For $p<p_{0}$, one may apply a standard interpolation argument (see, e.g. Chapter 4 of $[\mathbf{9} \mid$ ). In our argument, we need the factor $\eta_{2 R}$ given by the doubling property and get

$$
C_{1}(p)=\tilde{C}_{1}\left(p, N, \eta_{2 R}\right) C_{1}\left(p_{0}\right) \text {. }
$$

Note that $\eta_{2 R}$ only depends on $\sqrt{K} R$.

Proof of Theorem 1.5. As in the proof of Theorem 1.3 , by rescaling $u$ as

$$
\frac{u}{C_{0}\left[\inf _{B_{R / 2}} u+\mathcal{I}\left(f ; B_{R}, \eta_{2 R}\right)\right]},
$$


we may assume

$$
\inf _{B_{R / 2}} u+\mathcal{I}\left(f ; B_{R}, \eta_{2 R}\right) \leq \frac{1}{C_{0}} \quad \text { and } \quad \mathcal{I}\left(f ; B_{R}, \eta_{2 R}\right) \leq \delta_{0}
$$

By Theorem 1.3, we obtain

$$
\left(f_{B_{R / 2}} u^{p_{0}}\right)^{1 / p_{0}} \leq 1
$$

Using Lemma 10.1 and following the same argument as in the proof of Theorem 1.4. we obtain

$$
\sup _{B_{R / 2}} u \leq C_{1}\left(p_{0}\right)=C_{2} .
$$

The required estimate is achieved by re-scaling $u$ back.

11. Appendix: Fully-Nonlinear Uniform Elliptic Equations. In this appendix, we briefly explain how the proof presented herein could be extended to cover fully nonlinear uniform elliptic equations on Riemannian manifolds $\left(\nu=\operatorname{vol}_{g}\right)$. According to the standard theory of fully nonlinear PDEs, it suffices to prove the Harnack inequalities for the Pucci extremal operator.

Definition 11.1. Let $\theta \geq 1$ be a constant and $u \in C^{2}$. The Pucci extremal operator is defined by

$$
\begin{aligned}
\mathcal{M}_{\theta}^{-}[u](x) & :=\mathcal{M}_{\theta}^{-}(H):=\sum_{\lambda_{i} \geq 0} \lambda_{i}(H)+\theta \sum_{\lambda_{i} \leq 0} \lambda_{i}(H), \\
\mathcal{M}_{\theta}^{+}[u](x) & :=\mathcal{M}_{\theta}^{+}(H):=\sum_{\lambda_{i}<0} \lambda_{i}(H)+\theta \sum_{\lambda_{i} \geq 0} \lambda_{i}(H),
\end{aligned}
$$

where $H=\nabla^{2} u(x)$ and $\lambda_{i}(H)$ denote eigenvalues of $H$.

The next lemma demonstrates the generality and the extremity of the Pucci operator (see $[\mathbf{3}]$ ).

LEMMA 11.2. Let Sym $T \mathscr{M}$ be the bundle of $g$-self-adjoint operators on $T \mathscr{M}$. Let $H \in \operatorname{Sym} T \mathscr{M}$ be a section. Then

$$
\begin{aligned}
& \mathcal{M}_{\theta}^{-}(H):=\inf \{\operatorname{tr}(A \cdot H), A \in \operatorname{Sym} T \mathscr{M}, I d \leq A \leq \theta I d\} \\
& \mathcal{M}_{\theta}^{+}(H):=\sup \{\operatorname{tr}(A \cdot H), A \in \operatorname{Sym} T \mathscr{M}, I d \leq A \leq \theta I d\} .
\end{aligned}
$$

To study the Pucci operator, we introduce

$$
\mathcal{E}_{\theta}(r):=\sup _{(x, y)}\left\{\mathcal{M}_{\theta}^{+}\left(\nabla^{2}\left[\frac{1}{2} \rho_{y}^{2}\right](x)\right)-\operatorname{tr}\left(\nabla^{2}\left[\frac{1}{2} \rho_{y}^{2}\right](x)\right): \rho(x, y) \leq r\right\} .
$$

The following lemma proves some nice properties of this quantity. 
Lemma 11.3. Let $(M, g)$ be a complete Riemannian manifold. Then the following statements hold:

i) If $\nabla^{2}\left(\frac{1}{2} \rho_{y}^{2}\right)(x)$ is non-negative definite for all $x, y \in B_{R}$, then

$$
\mathcal{E}_{\theta}(2 R) \leq(\theta-1)(1+(n-1) \mathcal{H}(R \omega)),
$$

where $K$ is the Ricci lower bound in $B_{R}$. In particular, this holds for any ball $B_{R}$ with $R \leq R_{s}$, where $R_{s}$ depends on sectional curvature upper bound in $B_{2 R}$.

ii) Suppose, in $B_{R}$, the sectional curvatures are bounded from below by $-K_{s}$ with $K_{s} \geq 0$. Then

$$
\mathcal{E}_{\theta}(2 R) \leq(\theta-1)\left(1+(n-1) \mathcal{H}\left(\sqrt{K_{s} / n} R\right)\right) .
$$

Proof. The first part of i) follows immediately from the definition of $\mathcal{E}_{\theta}(R)$ and Ricci comparison. The second part of i) can be proved by applying the sectional curvature comparison against spheres. Statement ii) follows from standard sectional curvature comparison.

The proofs of Theorems 1.3 1.5 can be easily extended to prove the following Harnack inequalities for Pucci extremal operator.

THEOREM 11.4. Let $(\mathscr{M}, g, \nu)$ be a complete smooth Riemannian metricmeasure space. Let $K \geq 0$ be a constant. Let $u \in C^{2}\left(B_{2 R}\right) \cap C\left(\bar{B}_{2 R}\right)$ and $f \in C\left(B_{2 R}\right)$. Suppose that Ric $\left.\right|_{B_{2 R}} \geq-K g$. Then the following statments hold if we now assume $p_{0}, C_{1}, C_{2}$ (not including $\eta_{R}$ ) to depend on $R \sqrt{K}+\mathcal{E}_{\theta}(2 R)$ instead of $R \sqrt{K}$ :

i) $\mathcal{M}_{\theta}^{-}[u] \leq f$ and $u \geq 0$ in $B_{2 R}$ imply inequality (1.3);

ii) $\mathcal{M}_{\theta}^{+}[u] \geq f$ in $B_{2 R}$ implies inequality (1.4);

iii) $\mathcal{M}_{\theta}^{-}[u] \leq|f|$ and $\mathcal{M}^{+}[u] \geq f$ and $u \geq 0$ in $B_{2 R}$ imply inequality (1.5).

REMARK 11.5. In the case that $\left.S e c\right|_{B_{2 R}}=0$, Theorem 11.4 along with the Lemma 11.3 recovers the Harnack inequality proved in $[\mathbf{2}$ and $\mathbf{1 0}$.

REMARK 11.6. Unlike in the divergence case, if $\theta \neq 1$, we believe that the dependence on $\mathcal{E}_{\theta}(r)$ (hence sectional curvature) cannot be replaced by Ricci curvature. This can be seen in the following way. Fix some $x \in \mathscr{M}$, if the hessian $H(x):=\nabla^{2}\left(\frac{1}{2} \rho_{y}^{2}(x)\right)$ has a negative eigenvalue, then by choosing $\theta$ large,

$$
\mathcal{M}_{\theta}^{+}(H) \sim \theta \lambda_{n}(H), \quad \mathcal{M}_{\theta}^{-}(H) \sim \theta \lambda_{1}(H),
$$

where $\lambda_{1}, \lambda_{n}$ are, respectively, the least and greatest eigenvalue of $H$. Thus, $\mathcal{M}_{\theta}^{+}, \mathcal{M}_{\theta}^{-}$are affected by the extremal eigenvalues of $H$. While in divergence case the operator $\left(I_{n} \leq A \leq \theta I_{n}\right)$

$$
\int g\left(A \nabla \frac{\rho_{y}^{2}}{2}, \nabla \varphi\right) \sim \theta \rho_{y}|\nabla \varphi|, \quad \varphi \text { test function }
$$


is only affected by $\rho$. Based on this comparison, the dependence on $\mathcal{E}_{\theta}(R)$ seems necessary.

Proof of Theorem 11.4. Recall that the proofs in Sections 910 only rely on Lemma 8.1, hence it suffices to prove Lemma 8.1 for $\mathcal{M}_{\theta}^{-}$with $R \sqrt{K}$ in the constant $\mu, M$ replaced by

$$
R \sqrt{K}+\mathcal{E}_{\theta}(2 R)
$$

Reviewing the proof of Lemma 8.1, we see that it suffices to control $\Delta w$ from above on the contact set $A=A\left(a, B_{r / 6}\left(y_{0}\right) / B_{r}\left(x_{0}\right), w\right)$ (recall $\left.w=u+\psi\right)$ by $\mathcal{M}_{\theta}^{-} u \leq f$ and $R \sqrt{K}+\mathcal{E}_{\theta}$. This can be done as the following simple calculation shows.

For $x \in A$, by contact condition, we have $\nabla^{2} w(x) \geq-a \nabla^{2}\left(\frac{1}{2} \rho_{y}^{2}\right)(x)$. Denote $S=\nabla_{\nu}^{2} w(x)$ and $H=H_{y}^{\nu}(x)$. We then have $S+a H \geq 0$. Thus,

$$
\Delta u(x)=\operatorname{tr}[S+a H]-a \operatorname{tr}[H] \leq \mathcal{M}_{\theta}^{-}(S+a H)-a \operatorname{tr}[H] \leq \mathcal{M}_{\theta}^{-}(S)+a \mathcal{E}_{\theta}(2 r) .
$$

While $S=\nabla^{2} u+\nabla^{2} \psi$, by the elementary inequality regarding Pucci operator, we have

$$
\mathcal{M}_{\theta}^{-}(S) \leq \mathcal{M}_{\theta}^{-}[u](x)+\mathcal{M}^{+}[\psi](x) .
$$

Following the construction of the barrier $\psi$ (Lemma 6.4), one can easily see

$$
r^{2} \mathcal{M}^{+}[\psi] \leq-\alpha(\alpha+2)+a \mathcal{E}_{\theta}(2 r)
$$

Thus by replacing $\sqrt{K} R$ with $\sqrt{K} R+\mathcal{E}_{\theta}(R)$ in the expression of $\alpha, \Delta u$ is controlled on the contact set in the required way. The rest of the proof of Theorem 11.4 follows line by line from our earlier proofs in Sections 910 .

Note: The first version of this paper was posted on arXiv in 2011 (arXiv: 1102.5567). Right after that, we observed that the ABP estimate established in this paper can be used to give new proofs for some classical geometric inequalities. To point out this interesting geometric application of the ABP technique, we wrote a new paper $\mathbf{2 2}$, which included a version of the ABP estimate on standard Riemannian manifold and also briefly explained the application on Harnack inequalities to non-divergent equations. However, a detailed version of the ABP estimate on metric measure spaces and the Krylov-Safonov-Harnack inequalities for weighted Laplacian equation has never appeared. We would like to thank Professor Valentino Tosatti for his interest in this work and also encouraging us to publish this paper.

Acknowledgement: The authors would like to thank the anonymous referee for his/her extremely careful reading and useful comments, which improved the overall readability of the paper. 


\section{References}

1. Brighton K., A Liouville type theorem for smooth metric-measure spaces, Journal of Geometric Analysis, 23 (2013), Issue 2, 562-570.

2. Cabré X., Nondivergent elliptic equations on manifolds with nonnegative curvature, Comm. Pure Appl. Math., 50 (1997), 623-665.

3. Cabré X., Caffarelli L.A., Fully nonlinear elliptic equations, volume 43 of Colloquium Publications, AMS, 1995.

4. Caffarelli L.A., Interior $W^{2, p}$ estimates for solutions of the Monge-Ampére equation, Ann. of Math., 131(1) (1990), 135-150.

5. Calabi E., An extension of Hopf's maximum principle with an application to Riemannian geometry, Duke Math. J., 25 (1958), 45-56.

6. Cheeger J., Gromov M., Taylor M., Finite propagation speed, kernel estimates for functions of the Laplace operator, and the geometry of complete Riemannian manifolds, J. Differential Geometry, 17 (1982), 15-53.

7. Cheng S.Y., Li P., Yau S.-T., On the upper estimate of the heat kernel of a complete Riemannian manifold, Amer. J. Math., 103 (1981), 1021-1036.

8. Cheng S.Y., Yau S.-T., Differential equations on Riemannian manifolds and their geometric applications, Comm. Pure Appl. Math., 28 (1975), 333-354.

9. Han Q., Lin F., Elliptic Partial Differential Equations, Courant Lecture Notes. AMS/CIMS, 1997.

10. Kim S., Harnack inequality for nondivergent elliptic operators on Riemannian manifolds, Pacific J. Math., 213 (2) (2004), 281-293.

11. Krylov N.V., Safonov M.V., An estimate of the probability of a diffusion process hitting a set of positive measure, Dokl. Akad. Nauk SSSR, 245 (1979), 18-20.

12. Krylov N.V., Safonov M.V., A property of the solutions of parabolic equations with measurable coefficients, Izv. Akad. Nauk SSSR Ser. Mat., 44 (1980), 161-175.

13. Li X.D., Liouville theorems for symmetric diffusion operators on complete Riemannian manifolds, J. Math. Pures Appl., 84(9) (2005), 1295-1361.

14. Lott J., Some geometric properties of the Bakry-Émery Ricci tensor, Comment. Math. Helv, 78(4) (2003), 865-883.

15. Cordero-Erausquin D., McCann R., Schmuckenschlaeger M., A Riemannian interpolation inequality a la Borell, Brascamp and Lieb, Invent. Math., 146 (2001), 219-257.

16. Petersen P., Riemaniann Geometry, Number $\mathbf{1 7 1}$ in Graduate Texts in Mathematics. Springer, New York, second edition edition, 2006.

17. Qian Z.-M., Estimates for weight volumes and applications, J. Math. Oxford Ser., 48 (1987), 235-242.

18. Savin O., Small perturbation solutions for elliptic equations, Comm. Partial Differential Equations, 32 (2007), 557-578.

19. Varopoulos N., Hardy-littlewood theory for semigroups, J. Funct. Anal., 63 (1985), 240260.

20. Villani C., Optimal Transport: old and new, Springer-Verlag, Berlin, 2009.

21. Wang X. D., Zhang L., Local gradient estimate for p-harmonic functions on Riemannian manifolds, Communications in Analysis and Geometry, Volume 19(4) (2011), 759-771.

22. Wang Y., Zhang X.W., Alexandrov-Bakelman-Pucci estimate on Riemannian manifolds, Advance in Mathematics, 232(1) (2013), 499-512.

23. Wei G.F. and Wylie W., Comparison geometry for the Bakry-Émery Ricci tensor, J. Differential Geom., 83(2) (2009), 377-405. 
24. Yau S.-T., Harmonic functions on complete Riemannian manifolds, Comm. Pure Appl. Math., 28 (1975), 201-228.

25. Zhang Xi, Liouville type theorems for p-harmonic maps, Chinese Ann. Math. Ser. A, 21(1) (2000), 95-98.

Received June 29, 2018

Yu Wang

Department of Mathematics

Columbia University, New York, NY

e-mail: math.yuwang@gmail.com

Xiangwen Zhang

Department of Mathematics

University of California, Irvine

e-mail: xiangwen@math.uci.edu 